\title{
Diabetes Mellitus and Glucocorticoid Use As Risk Factors for COVID-19 Associated Mucormycosis
}

\section{Nasser Mikhail ${ }^{*}$, Soma Wali}

${ }^{1}$ Endocrinology Division, Olive-View-UCLA Medical Center, David-Geffen UCLA School of Medicine, Sylmar, CA, USA

${ }^{2}$ Department of Medicine, Olive-View-UCLA Medical Center, David-Geffen UCLA School of Medicine, Sylmar, CA, USA

Correspondence to: Nasser Mikhail, Endocrinology Division, Olive-View-UCLA Medical Center, David-Geffen UCLA School of Medicine, Sylmar, CA, USA.

Received date: July 2, 2021; Accepted date: July 13, 2021; Published date: July 19, 2021

Citation: Mikhail N, Wali S (2021) Diabetes Mellitus and Glucocorticoid Use As Risk Factors for COVID-19 Associated Mucormycosis. J Med Res Surg 2(4): pp. 1-4. doi: 10.52916/jmrs214051

Copyright: (C2021 Mikhail N, et al. This is an open-access article distributed under the terms of the Creative Commons Attribution License, which permits unrestricted use, distribution and reproduction in any medium, provided the original author and source are credited.

\section{ABSTRACT}

Background: A surge of cases of Coronavirus Disease 2019 (COVID-19)-Associated Mucormycosis (CAM) was recently observed.

Objective: To determine the contribution of diabetes and glucocorticoid therapy in predisposing to CAM. Methods: Pubmed search until July 2nd, 2021. Search terms included mucormycosis, diabetes, glucocorticoids, corticosteroids, coronavirus disease 2019, mortality. Randomized trials, case series, retrospective, pre-print studies, meta-analysis, professional guidelines are reviewed. Pertinent in vitro and animal studies are also included.

Results: Diabetes mellitus was reported in $78-85 \%$ of cases of CAM worldwide, with the highest rates present in India. Diabetic Ketoacidosis (DKA) was observed in $3.5-41 \%$ of cases of CAM. Glucocorticoid therapy emerged as another predisposing factor occurring in $85 \%$ of cases of CAM. Injudicious use of glucocorticoids may be a contributing factor in a substantial proportion of subjects with CAM. Majority of patients develop symptoms of CAM between day 10 and 15 from the diagnosis of COVID-19. However, some cases of CAM may present up to 3 months after COVID-19 or following recovery from COVID-19. Mortality rates of CAM overall ranges from $34-48 \%$. Surgical debridement may be associated with improved survival.

Conclusions: High index of suspicion for CAM should be present in patients with diabetes and those receiving corticosteroids. Effective glycemic control and judicious use of glucocorticoids should be implemented to decrease incidence of CAM.

\section{Keywords:}

Mucormycosis, COVID-19, Diabetes, Glucocorticoids, Corticosteroids, Dexamethasone, Mortality

\section{Introduction}

Shortly after the beginning of COVID-19, a surge of cases of CAM was observed worldwide. The largest number of cases of CAM was reported in a retrospective Indian study called Collaborative OPAI-IJO Study on Mucormycosis in COVID-19 (COSMIC). COSMIC included 2,826 patients with rhino-orbitalcerebral mucormycosis (ROCM) who were managed by ophthalmologists from January 1st 2020 to May 26, 2021 in 22 Indian states. The median (range) age was 51 (12-88) years. In the COSMIC, systemic use of corticosteroids and diabetes emerged as the 2 most common predisposing factors for CAM, being present in $87 \%$ and $78 \%$ of patients, respectively [1]. The largest international series of patients with CAM was published by Pal et al. [2], who described 99 cases until May 14, 2021. Again, most of these cases were reported from India (72\%), followed by the USA (10\%), and Egypt (6\%). Indeed, in preCOVID-19 era, it was already known that the highest prevalence of mucormycosis was present in India, close to 0.14 cases per 1000 population, a prevalence that is approximately 80 times that in developed countries [3]. In all reported series of CAM, there was a clear male preponderance. Thus, overall, $71-78 \%$ of patients with CAM were males $[1,2,4]$. The commonest causative species of mucormycosis was Rhizopus species $(85 \%$ of isolated species) [2].

Pathogenesis of Mucormycosis and Its Relevance to COVID-19, Hyperglycemia and Diabetic Ketoacidosis The main target of Mucorales, the fungus causing mucormycosis, is endothelial cells lining blood vessels [5]. Thus, this fungus invades endothelial cells after binding to a receptor located at the surface of endothelial cells called: glucose-related protein 78 (GRP78) [6]. Mucorales adhere to GRP78 receptors by expressing a spore Coat Homolog (CotH) protein [7]. Binding of the fungus CotH protein to the host receptor GRP78 leads to endocytosis of the organism and subsequent spread in the human body [5]. Free iron is essential for the survival of mucormycosis [8]. This is supported by the observation that iron starvation in vitro causes apoptosis of Rhizopus oryzae [9].

Importantly, COVID-19 infection is characterized by release of ferritin in the circulation as inflammatory marker [10]. As a result, ferritin loses part of its inner iron content leading to increased levels of free serum iron [10]. In addition, hyperglycemia may increase free iron by causing glycosylation of transferrin and ferritin thereby reduce iron binding [11]. Moreover, acidotic 
states, such as DKA, cause release of iron from iron-binding proteins such as transferrin [12]. Collectively, COVID-19 infection, hyperglycemia and DKA may therefore potentiate fungus virulence by increasing free iron availability. The elegant in vitro and in vivo studies in mice by the group of Gebrenmarian et al. [5], have clarified why patients with DKA are particularly predisposed to mucormycosis by recording the following observations. First, elevated albeit physiologic concentrations of glucose, free iron, and ketoacids, as prevailing in DKA, enhance expression of both the fungus ligand $\mathrm{CotH}$ and its receptor GRP78 [5]. Second, the enhanced expression of GRP78 and CotH were mainly driven by beta-hydroxy butyrate (representative of ketone bodies in DKA) and iron and to a lesser extent by glucose. Third, increased expressions of CotH and GRP78 were specific to beta-hydroxy butyrate since acidosis caused by lactic acid or HCL did not have any effect [5]. Fourth, beta-hydroxy butyrate abolished the ability of human neutrophils to kill the Rhizopus in cell culture [5]. Meanwhile, correction of acidosis by sodium bicarbonate completely reversed the inhibitory effect of beta-hydroxy butyrate on neutrophil function, and decreased fungus virulence and mortality in mice [5].

\section{Diabetes Mellitus As a Risk Factor for CAM}

Diabetes mellitus was present in $78-85 \%$ of cases of CAM $[1,2,4]$. Approximately $90 \%$ of CAM cases occurred in type 2 diabetes and $10 \%$ in type 1 diabetes [4]. These statistics are expected since diabetes was historically the most frequent underlying disease in mucormycosis in general. For instance, in the pre-COVID-19 era, the meta-analysis of Jeong et al. [13], showed that diabetes was the most common condition associated with mucormycosis occurring $40 \%$ of cases (340 of 851). Furthermore, diabetes emerged as independent risk factor for RCOM, odds ratio 2.49 (95\% Cl 1.77-3.54; $\mathrm{P}<0.001)$ [13]. The implication of diabetes in mucormycosis is particularly evident in low and middle-income countries $[3,13]$. Thus, in the international series reported by Hoengl et al. [4], diabetes was a more predominant risk factor for CAM in cases reported from India (40/42, 95.2\%). Most patients had uncontrolled diabetes with a median value of hemoglobin A1c (HbA1c) of $9.6 \%$. (range 4.8 to $17.1 \%$ ) [1]. The proportions of CAM patients having DKA varies widely being $3.5 \%$ in the Indian study by Sen et al. [1], $29 \%$ in the series of Pal et al. [2], and highest $41 \%$ in the global series reported by of Hoengl et al. [4]. This wide variation may be attributed in part to differences in definition of DKA and possibly reporting bias knowing that these studies are all retrospective in design $[1,2,4]$.

\section{Glucocorticoid Usage As Risk Factor for CAM}

Corticosteroids may facilitate mucormycosis infection by causing impairment of immunity, defective phagocytosis and worsening hyperglycemia [10]. Use of systemic glucocorticoids (oral or intravenous) was documented in $83-87 \%$ of patients with CAM $[1,2]$. In COSMIC, intravenous corticosteroids were given to $78 \%$ of patients for a median of 6 days [1]. Intravenous methylprednisolone (51\%) and dexamethasone (48\%) were the most commonly used agents [1]. Oral corticosteroids were used by $64 \%$ of patients for a median duration of 8 days [1]. In the international series reported by Pal et al. [2]. parenteral dexamethasone (dose and duration not mentioned) was the commonest form of administered glucocorticoid.

Interestingly, in the landmark RECOVERY Study from the UK, dexamethasone therapy has been shown to decrease mortality in patients with COVID-19 [14]. However, this mortality benefit was limited to COVID-19 patients with hypoxia, whereas in milder cases not requiring oxygen there was a trend towards increase mortality with dexamethasone therapy [14]. It should be emphasized that in the RECOVERY trial, dexamerthasone was used in small doses of $6 \mathrm{mg} / \mathrm{d}$ (equivalent to $32 \mathrm{mg} / \mathrm{d}$ of methylprednisolone, $40 \mathrm{mg} / \mathrm{d}$ of prednisone, or $160 \mathrm{mg} / \mathrm{d}$ of hydrocortisone) for up to 10 days [14]. The latter doses are clearly smaller than those implicated in predisposing to mucormycosis. Thus, a cumulative dose greater than $600 \mathrm{mg}$ of prednisone (equivalent to approximately $90 \mathrm{mg}$ of dexamethasone) has been found to predispose immunocompromised patients to mucormycosis [15]. Based on the above, corticosteroids should be used judiciously and only in hypoxic patients with COVID-19 who require oxygen support. Indeed, one factor that may have contributed to increased incidence of CAM in India was the inadvertent use of corticosteroids. Thus, data derived from COSMIC showed that despite the fact that 57\% (1602 of 2826) of patients required oxygen support, much more subjects $87 \%$ received corticosteroids [1]. In addition, 80\% (314 of 393) of hospitalized COVID-19 patients who did not need oxygen received corticosteroids [1]. Moreover, 21\% (373 of 1775) of patients received glucocorticoids more than the recommended duration of 10 days [1]. Interestingly, tocilizumab, another immunosuppressive agent used in severe forms of COVID-19 does not appear to predispose to mucormycosis since only $2 \%$ of patients with CAM were using this drug [1].

\section{Timing of CAM in Relation to Diagnosis of COVID-19}

Most cases of CAM occur concomitantly or shortly after diagnosis of COVID-19 [1]. The median time interval between COVID-19 diagnosis and first evidence of CAM diagnosis was 10-15 days $[2,4]$. However, few cases of CAM may occur up to 3 months after COVID-19 diagnosis $[1,16]$. Indeed, in the COSMIC, $44 \%$ of patients presented with CAM following recovery from COVID-19 [1]. This observation emphasizes the need for continued vigilance with respect to any symptoms or signs suggestive of CAM for up to 3 months after COVID-19 even after recovery from COVID-19.

\section{Prognosis of CAM}

Case series generally reported mortality rates of CAM ranging from $34 \%$ to $48.8 \%[1,4]$. However, the lowest mortality rates (14-37\%) were recorded in patients with ROCM, whereas the highest rates (81\%) were observed in the subgroup of patients with more invasive forms of mucormycosis including pulmonary, gastro-intestinal, and disseminated mucormycosis $[1,4]$. Median survival time from the day of diagnosis of mucormycosis was 75 days $(95 \% \mathrm{Cl}, 132.2-136.8)$ for patients with ROCM versus only 9 days $(95 \% \mathrm{Cl}, 2.3-15.7)$ for patients with pulmonary mucormycosis [4I]. Adjunctive surgery, used in $81 \%$ of patients, was associated with better survival in the series reported by Pal et al. $(\mathrm{P}<0.001)$ [2]. Similarly, in the series reported by Hongil et al. [4], mortality rates were lower in patients with ROCM who had adjunctive surgical treatment $(4 / 28,13.8 \%)$ versus patients treated by antifungals alone $(5 / 8$, $62.5 \%),(P=0.01)$. In the latter series, surgery did not impact 
the survival in patients with Central Nervous System (CNS) involvement [4]. Meanwhile, in COSMIC, there was evidence of improved survival in patients with ROCM invading the CNS who underwent surgical debridement of paranasal sinuses [1].

\section{Clinical Implications}

Since diabetes proved to be a common denominator in CAM, aggressive control of hyperglycemia is essential in patients with COVID-19. In cases complicated by DKA, rapid correction of acidosis is crucial given the role of ketoacidosis in aggravating mucormycosis infection [5]. Inadvertent use of glucocorticoids should be avoided in COVID-19 and limited to patients with hypoxia [14]. In fact, the roles of optimum glycemic control and appropriate use of glucocorticoids in decreasing incidence of CAM were supported by the absence of CAM cases in a large Indian series of 5,428 patients hospitalized with COVID-19 [17]. This achievement was accomplished by the implementation of strict protocols of glycemic control and use of low-dose corticosteroids [17]. Antifungal therapy should be rapidly initiated after consultation with the infectious disease service, In fact, delay in amphotericin therapy was shown to increase mortality among patients with hematologic malignancy affected by mucormycosis [18]. Surgical debridement should be considered for treatment of CAM since available retrospective data suggest that it may improve survival $[1,2,4]$. High index of suspicious for CAM should remain in effect for up to 3 months after diagnosis of COVID-19, particularly in patients with diabetes, DKA, and those using glucocorticoids.

\section{Conclusions and Current Needs}

Mucormycosis has been increasingly observed in association with COVID-19. Diabetes mellitus, with and without DKA, and use of corticosteroids emerged as the most common risk factors occurring in $78-87 \%$ of patients with CAM. Physicians should be aware of symptoms and signs of CAM to ensure timely diagnosis and treatment. In addition to strict glycemic control, judicious use of glucocorticoids, and hygienic hospital measures should be implemented [17]. Further studies are needed to determine the optimum prevention and treatment of CAM. Table 1 summarizes main measures for management of MAC based on available data.

Table 1: Measures for prevention and treatment of Coronavirus Disease 2019 (COVID-19)-Associated Mucormycosis (CAM).

\begin{tabular}{|l|l|}
\hline S. No. & Measures \\
\hline 1 & $\begin{array}{l}\text { Maintain a high index of suspicion regarding CAM, } \\
\text { particularly in patients with diabetes or receiving } \\
\text { glucocorticoids. }\end{array}$ \\
\hline 2 & $\begin{array}{l}\text { Be aware that CAM may occur up to } 3 \text { months after } \\
\text { onset of COVID-19. }\end{array}$ \\
\hline 3 & $\begin{array}{l}\text { Maintain hygienic hospital atmosphere to avoid } \\
\text { contamination by the fungus [17]. }\end{array}$ \\
\hline 4 & $\begin{array}{l}\text { Strict control of hyperglycemia, with optimum blood } \\
\text { glucose 140-180 mg/dl in hospitalized patients [19]. }\end{array}$ \\
\hline 5 & Rapid control of diabetic ketoacidosis. \\
\hline 6 & $\begin{array}{l}\text { Follow guidelines regarding corticosteroid indica- } \\
\text { tions, doses and duration of treatment [14]. }\end{array}$ \\
\hline
\end{tabular}

J Med Res Surg, OPEN 2 ACCESS

\begin{tabular}{|l|l|}
\hline 7 & $\begin{array}{l}\text { Early administration of anti-fungal therapy in case of } \\
\text { suspicion of CAM. }\end{array}$ \\
\hline 8 & $\begin{array}{l}\text { Surgical debridement when indicated in addition to } \\
\text { anti-fungal therapy. }\end{array}$ \\
\hline
\end{tabular}

Conflict of Interest: The authors do not have any conflict of interest to declare.

\section{Funding: None.}

\section{References}

1. Sen M, Honavar SG, Bansal R, et al. (2021) Epidemiology, clinical profile, management, and outcome of COVID-19associated rhino-orbital-cerebral mucormycosis in 2826 patients in India - Collaborative OPAI-IJO Study on Mucormycosis in COVID-19 (COSMIC), Report 1. Indian J Ophthalmology 69(7): pp. 1670-1692.

2. Pal R, Singh B, Bhadada SK, et al. (2021) COVID-19-associated mucormycosis: An updated systematic review of literature. Mycoses. Epubhead of print.

3. Skiada A, Pavleas I (2020) Drogari-Apiranthitou M. Epidemiology and Diagnosis of Mucormycosis: An Update. J Fungi (Basel) 6(4): p. 265.

4. Hoenigl M, Seidel D, Carvalho A, et al. (2021) The Emergence of COVID-19 associated mucormycosis: Analysis of cases From 18 Countries.

5. Gebremariam T, Lin L, Liu M, et al. (2016) Bicarbonate correction of ketoacidosis alters host-pathogen interactions and alleviates mucormycosis. J Clin Invest 126(6): pp. 2280-2294.

6. Liu M, Spellberg B, Phan QT, et al. (2010) The endothelial cell receptor GRP78 is required for mucormycosis pathogenesis in diabetic mice. J Clin Invest 120(6): pp. 1914-1924.

7. Gebremariam T, Liu M, Luo G, et al. (2014) CotH3 mediates fungal invasion of host cells during mucormycosis. J Clin Invest 124(1): pp. 237-250.

8. Petrikkos G, Tsioutis C (2018) Recent Advances in the Pathogenesis of Mucormycoses. Clin Ther 40(6): pp. 894-902.

9. Shirazi F, Kontoyiannis DP, Ibrahim AS (2015) Iron starvation induces apoptosis in Rhizopus oryzae in vitro. Virulence. 6(2): pp. 121-126.

10. Jose A, Singh S, Roychoudhury A, et al. (2021) Current Understanding in the Pathophysiology of SARS-CoV-2-Associated Rhino-Orbito-Cerebral Mucormycosis: A Comprehensive Review. J Maxillofac Oral Surg 16: pp. 1-8.

11. Morales-Franco B, Nava-Villalba M, Medina-Guerrero EO, et al. (2021) Host-Pathogen Molecular Factors Contribute to the Pathogenesis of Rhizopus spp. in Diabetes Mellitus. Curr Trop Med Rep: pp. 1-12.

12. Abe F, Shibuya H, Tateyama M, et al. (1986) Mucormycosis in diabetic ketoacidosis. Role of unbound iron binding capacity of transferrin. Acta Pathol Jpn 36(10): pp. 1507-1512.

13. Jeong W, Keighley C, Wolfe R, et al. (2019) The epidemiology and clinical manifestations of mucormycosis: a systematic review and meta-analysis of case reports. Clin Microbiol Infect 25(1): pp. 26-34.

14. RECOVERY Collaborative Group (2021) Dexamethasone in 
Hospitalized Patients with Covid-19. N Engl J Med 384(8): pp. 693-704.

15. Lionakis MS, Kontoyiannis DP (2003) Glucocorticoids and invasive fungal infections. Lancet 362(9398): pp. 1828-1838.

16. Khatri A, Chang KM, Berlinrut I, et al. (2021) Mucormycosis after Coronavirus disease 2019 infection in a heart transplant recipient - Case report and review of literature. J Mycol Med 31(2): p. 101125.

17. Mulakavulupil B, Vaity C, Joshi S, et al. (2021) Absence of case of mucormycosis (March 2020-May 2021) under strict protocol driven management care in a COVID-19 specific tertiary care intensive care unit. Diab Metab Synd Clin Res Rev 15(4): p. 102169.

18. Chamilos G, Lewis RE, Kontoyiannis DP (2008) Delaying amphotericin B-based frontline therapy significantly increases mortality among patients with hematologic malignancy who have zygomycosis. Clin Infect Dis 47(4): pp. 503-509.

19. American Diabetes Association (2021) Diabetes care in the hospital. Diabetes Care 44 (Supplement 1): S211-S220. 